\title{
Membrane versus centrifuge-based therapeutic plasma exchange: a randomized prospective crossover study
}

\author{
Carsten Hafer $^{1} \cdot$ Paulina Golla $^{1} \cdot$ Marion Gericke $^{2} \cdot$ Gabriele Eden $^{1,4}$. \\ Gernot Beutel $^{3} \cdot$ Julius J. Schmidt $^{1} \cdot$ Bernhard M. W. Schmidt $^{1} \cdot$ Stef De Reys $^{2}$ • \\ Jan T. Kielstein ${ }^{1,4}$
}

Received: 27 July 2015 / Accepted: 9 October 2015 / Published online: 3 November 2015

(C) Springer Science+Business Media Dordrecht 2015

\begin{abstract}
Background Therapeutic plasma exchange (TPE) is either performed using a highly permeable filter with standard multifunctional renal replacement equipment (MTPE) or a centrifugation device (cTPE). Although both techniques are well established in clinical practice, performance of these two modes of TPE was never compared in a prospective randomized fashion. Thus we aimed to compare two commercially available therapeutic apheresis systems: mTPE (Octonova with Plasmaflo filter) and cTPE (Spectra Optia apheresis system).

Methods Twenty-one patients (age $51.6 \pm 13.5$ years; 10 $\mathrm{F} / 11 \mathrm{M}$; BMI $25.1 \pm 5.0 \mathrm{~kg} / \mathrm{m}^{2}$ ) were enrolled in this randomized, prospective, paired, crossover study performed in the Hannover Medical School, Germany. First treatment (either mTPE or cTPE) was chosen by an online randomization list. The primary endpoints were plasma removal efficiency with $1.2 \times$ of the total plasma volume exchanged. Secondary endpoints were total amount of plasma substances removed, such as IgG and fibrinogen. Further, the
\end{abstract}

Carsten Hafer and Paulina Golla have contributed equally to this work.

Jan T. Kielstein

Kielstein@yahoo.com

1 Department of Nephrology and Hypertension, Medical School Hannover, Carl-Neuberg-Strasse 1, 30625 Hannover, Germany

2 Terumo BCT, Zaventem, Belgium

3 Department of Haematology, Oncology and Stem Cell Transplantation, Medical School Hannover, Hannover, Germany

4 Medical Clinic V, Teaching Hospital Braunschweig, Braunschweig, Germany treatment effect on platelet count and complications were evaluated.

Results Despite a comparable volume of the processed plasma, mTPE treatment time was $10.5 \%$ longer than cTPE treatment time $(p<0.05)$, resulting in a $10 \%$ lower plasma removal rate of the mTPE treatment. Both treatments were comparable in terms of decrease in median (IQR) IgG [pre-mTPE 5.34 (3.48-8.37), post-mTPE 1.96 (1.43-2.84) g/L; pre-cTPE 5.88 (3.42-8.84), post-cTPE $1.89(1.21-3.52) \mathrm{g} / \mathrm{L}]$. Also the median (IQR) amount of IgG removed in mTPE [13.14 (7.42-16.10) g] was not different from the cTPE treatment [9.30 (6.26-15.69) g]. This was also true for IgM removal. Platelet loss during mTPE was nearly twice as much as with cTPE $(15 \pm 9$ versus $7 \pm 9 \%, p<0.05)$.

Conclusion Although the centrifugal procedures were conducted using flow rates that could easily be obtained using peripheral access, plasma removal efficiency was significantly higher and treatment time was significantly lower in cTPE as compared to MTPE. Despite this lower treatment time, the decline in markers of procedure efficacy was comparable. Especially in centers performing many procedures per year, cTPE in contrast to MTPE can reduce treatment time without compromising treatment efficacy.

Keywords Antibodies $\cdot \operatorname{IgG} \cdot$ Extracorporeal treatment . Apheresis

\section{Introduction}

Therapeutic plasma exchange (TPE) is an extracorporeal blood purification technique that was first described in 1914 [1]. It removes pathogenic substances such as autoantibodies, lipoproteins and circulating immune complexes from 
the plasma [9] and plays a key role in the management of various diseases. According to the 2013 guidelines of the American Society of Apheresis, it is the treatment of choice for acute ANCA-associated rapid progressive glomerulonephritis, thrombotic thrombocytopenic purpura, GuillianBarré syndrome, Goodpasture's syndrome and cardiac allograft rejection [13]. Especially in the transplant setting and in patients with neurological disease, treatment numbers are increasing $[5,11]$. TPE continues to be used in indications being far from established like during the 2011 German STEC-HUS crisis [6] or in sepsis [4]. At our institution alone, the number of annual TPEs increased from 190 treatments in 2004 to 1215 treatments in 2014. There are two well-established modes to perform TPE using either a highly permeable filter with (mTPE) in conjunction with a multifunctional hemofiltration/dialysis device or a centrifugation device (cTPE) whose operation mode is limited to apheresis and cell collection. It is viewed as the preferred mode for the treatment of septic patients [14]. While mTPE is preferred by nephrology departments as a management option in the treatment of autoimmune-related renal diseases such as microangiopathic hemolytic anemias, cTPE is preferentially used in transfusion medicine for a variety of diseases in the field of nephrology, neurology and hematology. According to the 2007 International Apheresis Registry, surveys of apheresis have shown geographical differences of clinical practice and the type of technologies used [7]. Plasma treatment by membrane filtration is preferred both in Germany and Japan. By contrast, in the USA, TPE is primarily performed by centrifugation. Even though the differences between MTPE and cTPE had been extensively discussed in review papers over the last decades [3, 16], a head-to-head comparison of these two techniques in regard to the duration of treatment and effectiveness of separation does so far not exist. Thus, we designed a randomized prospective trial to test effectiveness of both techniques to perform the first ever controlled comparison of both techniques.

\section{Patients and methods}

The study was approved by the local ethics committee of Hannover Medical School, Germany, protocol \#5343. All patients gave written informed consent before enrollment into the study.

\section{Study design}

This was a prospective, open-label, randomized, crossover study to compare mTPE using the OctoNova (Diamed, Cologne, Germany) device with the Plasmaflo OP 05W (Asahi Kasei Medical, Japan) filter to cTPE using the Spectra Optia (Terumo BCT, Lakewood, CO, USA) apheresis device. The study setting was the critical care nephrology unit of a tertiary care hospital.

\section{Study protocol}

This study included 21 patients with the need for TPE as reported elsewhere [12]. The inclusion criteria were:

(1) Indication for TPE; (2) age between 18 and 80 years; and (3) written informed consent. Exclusion criteria were: (1) need for fresh-frozen plasma as replacement fluid and (2) participation in another study. Albumin solution was individually and freshly made up immediately before TPE by mixing $20 \%$ albumin solution $(100 \mathrm{ml}$ bottles $)$ and Duosol $^{\circledR}$ a ready-to-use bicarbonate dialysate solution (B. Braun, Melsungen, Germany) containing $2 \mathrm{mmol} / \mathrm{L}$ potassium to yield the calculated volume of replacement fluid.

The device for the first treatment was allocated by randomization (automatically online generated). After the first TPE either with membrane or centrifuge technique, the second TPE was done in a crossover design, i.e., switch to the other technique. For patients who underwent more than two TPEs, only the first two treatments were recorded. Patient selection was restricted to those that received albumin as replacement fluid, to allow the measurement of plasma constituents in the removed plasma. All but one patient had central venous access. One had a native arteriovenous fistula.

Laboratory markers analyzed were: hematocrit, hemoglobin, red and white blood cell count, platelet count, CH50, plasma fibrinogen as well as plasma $\operatorname{IgG}$ and $\operatorname{IgM}$. All measurements were performed using routine laboratory tests using certified assay methods.

The following parameters were calculated: total blood volume $(\mathrm{TBV})(\mathrm{TBV}=$ weight $\times 70 \mathrm{~mL} / \mathrm{kg}$ for male resp. weight $\times 65 \mathrm{~mL} / \mathrm{kg}$ for female) and total plasma volume (TPV) $(\mathrm{TPV}=\mathrm{TBV} \times 1$-hct), which was used for all further analysis. The TPV was once compared with the TPV calculated by the Kaplan formula.

Processing time, plasma volume exchanged, blood flow, transmembrane pressure and removal bag volume were recorded. The reduction in the examined biomarkers (comparing pre- and post-TPE levels) was done by drawing the first sample from the vascular access site immediately before the start of treatment. Post-TPE samples were drawn from the vascular access site before rinse back was done. In addition we took samples from the removed and collected plasma in the waste bag to allow the measurement of the total removed biomarkers.

\section{Statistical analysis}

Data are presented as median and interquartile range (IQR). Comparisons between both techniques and between preand post-TPE laboratory marker levels were performed 
using a paired $t$ test. Differences were considered to be statistically significant if $p$ values were $<0.05$.

\section{Results}

The average age of the patients was $51.6 \pm 13.5$ years. Ten $(47.6 \%)$ of them were females. The average BMI was $25.1 \pm 5.0 \mathrm{~kg} / \mathrm{m}^{2}$. Underlying diseases necessitating TPE were humoral rejection after solid organ transplantation $(n=8)$; Guillian-Barré syndrome and variants $(n=3)$; monoclonal gammopathy $(n=2)$; multiple sclerosis $(\mathrm{n}=2)$; microscopic polyangiitis $(n=2)$; rapid progressive glomerulonephritis $(n=1)$; cryoglobulinemia $(n=1)$; and cytotoxic antibodies $(n=1)$. Further patients' characteristics and details of the procedure are described elsewhere [15].

Out of the 21 patients, 20 could be retained for final analysis. One patient dropped out as only one treatment was performed. In the final 20 patients, 40 therapeutic TPEs were included in the final analysis. All treatments were well tolerated by the patients.

The median (IQR) total plasma volume based on the Nadler-Allen formula prior to the MTPE treatment was 3045 (2771-3527) $\mathrm{mL}$ and did not differ from the plasma volume prior to the cTPE treatment where it was 2979 (2752-3502) $\mathrm{mL}$. Also the use of the Kaplan formula could not detect a difference in plasma volume between mTPE [3405 (2735-3820) $\mathrm{mL}$ ] and the cTPE treatment [3229 (2676-3872) $\mathrm{mL}]$.

Treatment time of the mTPE treatment was significantly longer $132.5(125.0-148.8) \mathrm{min}$ than the cTPE treatment [120.0 (114.3-138.0) min], Fig. 1a. This resulted in a lower plasma removal rate of the MTPE treatment 27.00 (25.2528.75) $\mathrm{mL} / \mathrm{min}$ than during the cTPE treatment $[30.00$ (26.25-32.50) $\mathrm{mL} / \mathrm{min}$ Fig. 1b]. The processed blood volume required to remove $1.2 \times$ total plasma volume in mTPE procedures was significantly higher than with cTPE $(19.86 \pm 3.42$ versus $6.46 \pm 1.23 \mathrm{~L}, p<0.05)$. As a result, the plasma removal efficiency was significantly higher in cTPE than in mTPE ( $84 \pm 11$ versus $27 \pm 5 \%, p<0.05)$. Both treatments were comparable in terms of decrease in $\operatorname{IgG}$ and $\operatorname{IgM}$ (Fig. 2). Also, the absolute amount of $\operatorname{IgG}$ and IgM was removed in mTPE (Fig. 2).

During the MTPE treatment, one circuit failure occurred, making a change of the system necessary. In another patient, a preemptive system change was performed due to an increase in TMP. The TMP in the mTPE group increased significantly from $14.0(9.0-19.5)$ to $21.0(13.5-26.0)$ $\mathrm{mm} \mathrm{Hg}(p=0.048)$. For the cTPE treatment, citrate was used as an anticoagulant with an inlet/AC ratio of 10/1. In these patients, we did not detect a difference in the ionized calcium comparing the start and the end of treatment
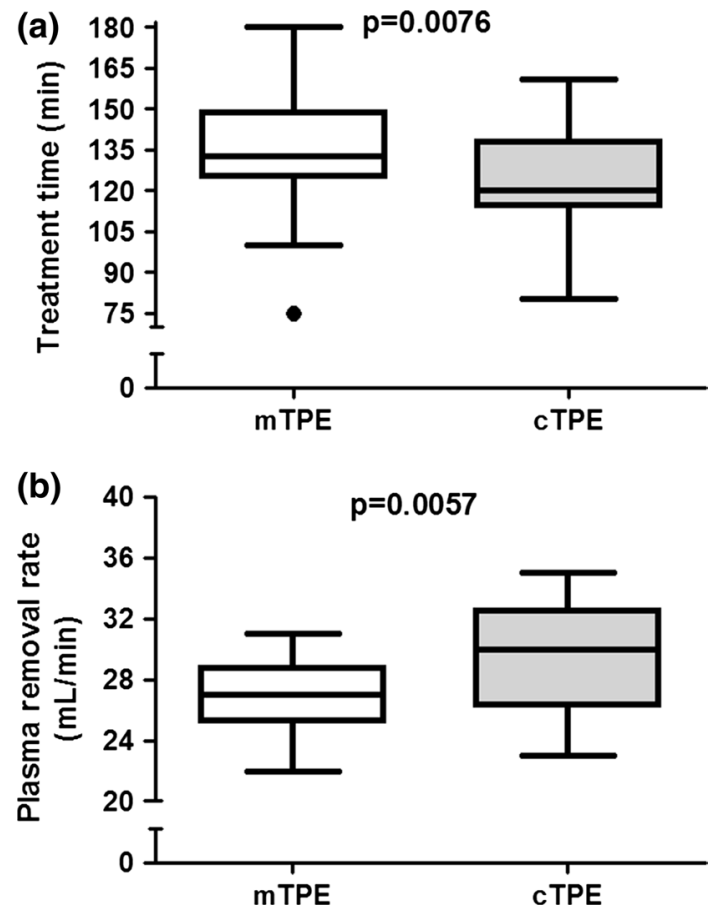

Fig. 1 a Procedure time during the mTPE and cTPE treatment. b Plasma removal rate during the MTPE and cTPE treatment

$\left[\begin{array}{lllll}1.17 & (1.13-1.21) & \text { versus } & 1.13 & (1.08-1.18) \\ \mathrm{mmol} / \mathrm{L} \text {; }\end{array}\right.$ $p=0.126]$. For mTPE, unfractionated heparin was used. Patients received a bolus injection of $2000 \mathrm{IU}$ followed by a $1000 \mathrm{IU} / \mathrm{h}$ thereafter. Platelet loss during $\mathrm{mTPE}$ was nearly twice as high as with cTPE $(15 \pm 9$ versus $7 \pm 9 \%$, $p<0.05$ ) (Table 1). While both hemoglobin level and hematocrit remained unchanged in the MTPE treatment, there was a slight but significant increase in both parameters in the cTPE-treated patients (Table 1), possibly due to a hemoconcentration in the latter group. However, we do not have a patient weight before the rinse back, i.e., the time the blood sample was drawn.

\section{Discussion}

The pertinent findings of our study were that cTPE as compared to MTPE provided a comparable treatment quality in a shorter period of time. To our knowledge, this is the first study directly comparing cTPE and mTPE.

\section{Treatment time}

Treatment time of mTPE was $10.4 \%$ longer than for cTPE. Even though a median 13-min difference in treatment time can be almost neglected in centers that only rarely perform TPE, for high-volume centers like ours the amount of saved nursing time is substantial, in our case roughly $270 \mathrm{~h}$ per 

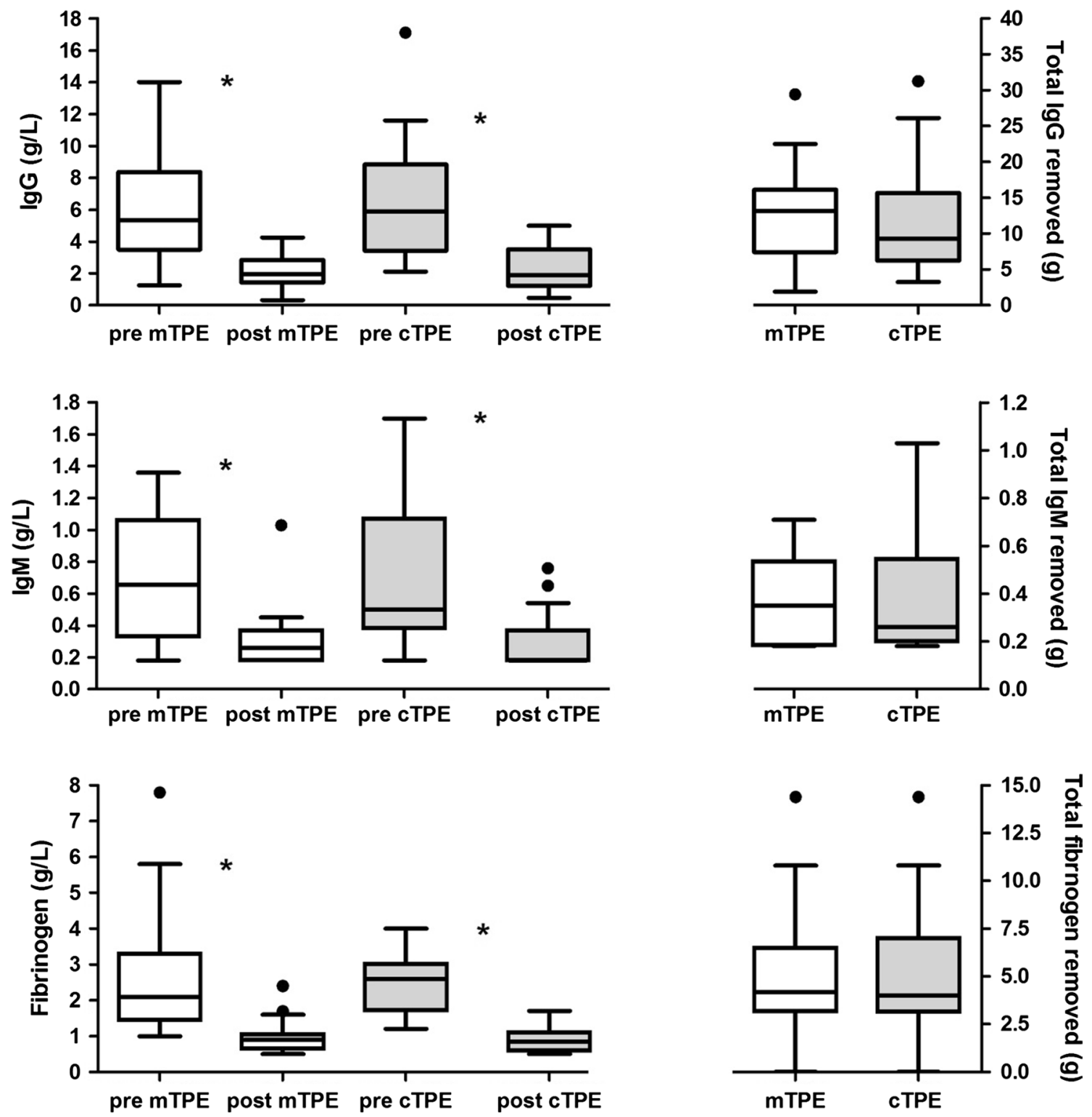

Fig. 2 Pre- and post-procedure plasma levels of $\operatorname{IgG}$ and fibrinogen and the corresponding total amount of these compounds in the removed plasma. Data are visualized as box-and-whisker plots (horizontal bars indicate median values)

Table 1 Laboratory parameters before and after mTPE and cTPE

\begin{tabular}{llcccc}
\hline Parameter & Unit & MTPE before & MTPE after & CTPE before & CTPE after \\
\hline Hematocrit & $\%$ & $33.90(31.08-37.80)$ & $32.79(28.83-37.65)$ & $33.85(30.33-37.98)$ & $34.95 *(30.93-38.43)$ \\
Hemoglobin & $\mathrm{g} / \mathrm{dL}$ & $11.30(10.03-11.98)$ & $10.90(9.45-12.15)$ & $11.10(10.03-12.23)$ & $11.50 *(10.23-12.28)$ \\
PLT & $\mathrm{Mill} / \mathrm{L}$ & $237.5(176.3-310.0)$ & $209.5 *(141.5-254.5)$ & $235.5(174.0-284.8)$ & $222.0 *(151.0-293.0)$ \\
Leukocytes & $10^{3} / \mu \mathrm{L}$ & $8.00(3.92-11.43)$ & $8.55^{*}(5.47-13.3)$ & $6.70(4.82-13.03)$ & $7.30 *(5.37-13.95)$
\end{tabular}

$* p<0.05$ comparing pre-and post-TPE values per treatment mode

year. This even holds true despite the fact that we kept the blood inlet flow in a range that is frequently observed in patients in whom peripheral veins are used for access.
Hence the time saved by using cTPE instead of mTPE could have been even more substantial if the maximum blood flow in the central venous access allowed would have 
been used. Therefore, it is very likely that in patients with a central line using higher inlet flow more cTPE would require even less time, i.e., would result in more substantial reduction in treatment time as compared to $\mathrm{MTPE}$, a finding of possible relevance to centers performing $>50-100$ procedures a year, especially given the limited human and financial resources in the medical system.

\section{Treatment quality}

Aside from the treatment time, we investigated several parameters of treatment quality such as removal of $\mathrm{IgG}$, IgM and fibrinogen as well as the effect of treatment modality on platelets and leukocytes. None of those parameters showed a marked difference. It is worthwhile to point out that this especially holds true for the platelet count, which decreased less in cTPE, arguing against a negative effect of cTPE which had been found three decades ago in cTPE [2]. Even though our study was not powered to compare incidence of circuit failure, we did not observe a circuit failure during the cTPE treatments. The underlying reason might have been the routine use of citrate anticoagulation, whose main advantage is the reduction in bleeding risk [8]. Other authors have even attributed a modulating effect on the immunes system with the use of citrate [10]. In a subgroup of 10 patients, we could, however, not detect a different effect on the complement system using the CH50 test. It is important to point out that none of the CTPE patients experienced clinical or laboratory side effects, i.e., hypocalcemia. By contrast, during the mTPE treatment we observed one circuit clotting. Two circuits had been preemptively changed due to a rise in transmembrane pressure. This potential difference in circuit failure rate between cTPE and $\mathrm{mTPE}$ should be investigated in larger studies. Moreover, it would be of interest to elucidate which role the sole use of unfractionated heparin has in this regard.

\section{Limitations of the study}

There are several limitations of our study that we wish to point out. We performed a single-center study in a limited number of patients. Nonetheless, due to the crossover design of our study, every patient served as his/her own control. Moreover, we did not use peripheral veins as access, an access site frequently used in the outpatient setting, yet our inlet flow is in the range frequently used in this setting, and thus, our data are representative of this patient population. On the other hand, we did not use the maximum inlet flow that would have been possible with a central venous access, thus representing a bias underestimating the possible performance of the cTPE. Further, we only analyzed treatment time, but not time for machine set-up and related activities. Lastly, our study was neither aimed nor powered to evaluate procedure-related complications, let alone clinical outcome. In summary, especially for units performing frequent TPEs, cTPE with its shorter treatment might be the preferred mode over mTPE, yet other factors such as technical experiences with the techniques, learning curve of the staff as well as off-hour procedures and the price of disposables also impact this decision.

Acknowledgments The investigator-initiated study was supported by an unrestricted grant from Terumo BCT.

\section{Compliance with ethical standards}

Conflict of interest JTK received research grants from Fresenius Medical Care and Diamed as well as speaker fees from Fresenius Medical Care and Diamed. All other authors declare that they have no conflict of interest.

Ethical approval The study was approved by the Ethical Board of the Hannover Medical School (protocol \# 5343). All procedures performed in studies involving human participants were in accordance with the ethical standards of the institutional and/or national research committee and with the 1964 Helsinki Declaration and its later amendments or comparable ethical standards. Written informed consent was obtained from all individual participants included in the study.

\section{References}

1. Abel JL, Rowntree LG, Turner BB (1914) Plasma removal with return of corpuscles (plasmaphaeresis). J Pharmacol Exp Ther 5:625-641

2. Fosburg M, Dolan M, Propper R, Wolfe L, Kevy S (1983) Intensive plasma exchange in small and critically ill pediatric patients: techniques and clinical outcome. J Clin Apher 1:215-224

3. Gurland HJ, Lysaght MJ, Samtleben W, Schmidt B (1984) A comparison of centrifugal and membrane-based apheresis formats. Int J Artif Organs 7:35-38

4. Hadem J, Hafer C, Schneider AS, Wiesner O, Beutel G, Fuehner T, Welte T, Hoeper MM, Kielstein JT (2014) Therapeutic plasma exchange as rescue therapy in severe sepsis and septic shock: retrospective observational single-centre study of 23 patients. BMC Anesthesiol 14:24

5. Ius F, Sommer W, Tudorache I, Kuhn C, Avsar M, Siemeni T, Salman J, Hallensleben M, Kieneke D, Greer M, Gottlieb J, Kielstein JT, Boethig D, Welte T, Haverich A, Warnecke G (2015) Preemptive treatment with therapeutic plasma exchange and rituximab for early donor-specific antibodies after lung transplantation. J Heart Lung Transplant 34:50-58

6. Kielstein JT, Beutel G, Fleig S, Steinhoff J, Meyer TN, Hafer C, Kuhlmann U, Bramstedt J, Panzer U, Vischedyk M, Busch V, Ries W, Mitzner S, Mees S, Stracke S, Nurnberger J, Gerke P, Wiesner M, Sucke B, Abu-Tair M, Kribben A, Klause N, Schindler R, Merkel F, Schnatter S, Dorresteijn EM, Samuelsson O, Brunkhorst R (2012) Best supportive care and therapeutic plasma exchange with or without eculizumab in Shiga-toxin-producing E. coli O104:H4 induced haemolytic-uraemic syndrome: an analysis of the German STEC-HUS registry. Nephrol Dial Transplant 27:3807-3815

7. Malchesky PS, Koo AP, Skibinski CI, Hadsell AT, Rybicki LA (2010) Apheresis technologies and clinical applications: the 2007 International Apheresis Registry. Ther Apher Dial 14:52-73 
8. Mehta RL, McDonald BR, Aguilar MM, Ward DM (1990) Regional citrate anticoagulation for continuous arteriovenous hemodialysis in critically ill patients. Kidney Int 38:976-981

9. Okafor C, Ward DM, Mokrzycki MH, Weinstein R, Clark P, Balogun RA (2010) Introduction and overview of therapeutic apheresis. J Clin Apher 25:240-249

10. Oudemans-van Straaten HM, Kellum JA, Bellomo R (2011) Clinical review: anticoagulation for continuous renal replacement therapy-heparin or citrate? Crit Care 15:202

11. Schiffer M, Kielstein JT (2011) ABO-incompatible renal transplantation: from saline flushes to antigen-specific immunoadsorption-tools to overcome the barrier. Korean J Hematol 46:164-168

12. Schmidt JJ, Jahn J, Golla P, Hafer C, Kielstein JT, Kielstein H (2015) Effect of therapeutic plasma exchange on plasma levels and total removal of adipokines and inflammatory markers. BMC Obes 2:37
13. Schwartz J, Winters JL, Padmanabhan A, Balogun RA, Delaney M, Linenberger ML, Szczepiorkowski ZM, Williams ME, Wu Y, Shaz BH (2013) Guidelines on the use of therapeutic apheresis in clinical practice-evidence-based approach from the Writing Committee of the American Society for Apheresis: the sixth special issue. J Clin Apher 28:145-284

14. Stegmayr BG, Jakobson S, Rydvall A, Bjorsell-Ostling E (1995) Plasma exchange in patients with acute renal failure in the course of multiorgan failure. Int J Artif Organs 18:45-52

15. Tutarel O, Golla P, Beutel G, Bauersachs J, David S, Schmidt BM, Lichtinghagen R, Kielstein JT (2012) Therapeutic plasma exchange decreases levels of routinely used cardiac and inflammatory biomarkers. PLoS One 7:e38573

16. Ward DM (2011) Conventional apheresis therapies: a review. J Clin Apher 26:230-238 